\title{
Charge transport in InAs nanowire Josephson junctions
}

\author{
Simon Abay, ${ }^{1, *}$ Daniel Persson, ${ }^{1}$ Henrik Nilsson, ${ }^{2}$ Fan Wu, ${ }^{1}$ H. Q. Xu, ${ }^{2,3}$ Mikael Fogelström, ${ }^{1}$ \\ Vitaly Shumeiko, ${ }^{1}$ and Per Delsing ${ }^{1, \dagger}$ \\ ${ }^{1}$ Department of Microtechnology and Nanoscience (MC2), Chalmers University of Technology, SE-412 96 Göteborg, Sweden \\ ${ }^{2}$ Division of Solid State Physics, Lund University, Box 118, S-221 00 Lund, Sweden \\ ${ }^{3}$ Key Laboratory for the Physics and Chemistry of Nanodevices and Department of Electronics, Peking University, Beijing 100871, China
}

(Received 8 November 2013; revised manuscript received 16 May 2014; published 23 June 2014)

\begin{abstract}
We present an extensive experimental and theoretical study of the proximity effect in InAs nanowires connected to superconducting electrodes. We fabricate and investigate devices with suspended gate-controlled nanowires and nonsuspended nanowires, with a broad range of lengths and normal-state resistances. We analyze the main features of the current-voltage characteristics: the Josephson current, excess current, and subgap current as functions of length, temperature, magnetic field, and gate voltage, and compare them with theory. The Josephson critical current for a short-length device, $L=30 \mathrm{~nm}$, exhibits a record high magnitude of $800 \mathrm{nA}$ at low temperature that comes close to the theoretically expected value. The critical current in all other devices is typically reduced compared to the theoretical values. The excess current is consistent with the normal resistance data and agrees well with the theory. The subgap current shows a large number of structures; some of them are identified as subharmonic gap structures generated by multiple Andreev reflection. The other structures, detected in both suspended and nonsuspended devices, have the form of voltage steps at voltages that are independent of either the superconducting gap or length of the wire. By varying the gate voltage in suspended devices, we are able to observe a crossover from typical tunneling transport at large negative gate voltage, with suppressed subgap current and negative excess current, to pronounced proximity junction behavior at large positive gate voltage, with enhanced Josephson current and subgap conductance as well as a large positive excess current.
\end{abstract}

DOI: 10.1103/PhysRevB.89.214508

PACS number(s): 74.45.+c, 74.50.+r, 74.25.F-

\section{INTRODUCTION}

Semiconducting nanowires (NW) have been a focus of intensive research for their potential applications as building blocks in nanoscale devices [1-4]. The nanoscale dimension of the semiconducting nanowires, comparable to the electronic Fermi wavelength, also makes them an attractive platform for studying the fundamental phenomena of quantum transport. By tuning the Fermi wavelength by means of electrostatic gates, one gets access to such quantum phenomena as conductance quantization [5,6], and quantum interference effects [7].

Another research interest has been the proximity effect in nanowires induced by connecting them to superconducting electrodes (S) [8,9]. In such devices, S-NW-S, the nanowire serves as a weak link through which a supercurrent can flow due to the presence of the phase difference between the superconducting condensates [9-11].

Among a variety of nanowires tested in experiments, nanowires of InAs play a central role $[8,10]$. This is due to their material properties: high electron mobility, low effective mass, and pinning of the Fermi level in the conduction band that permits highly transparent galvanic S-NW contacts. Hybrid devices of InAs nanowires have demonstrated Andreev subgap conductance [6,12,13], Josephson field effect [9,10], and Cooper-pair beam splitting [14]. More recently, the nanowire hybrid devices attracted new attention following theoretical predictions of Majorana bound states in NW-S proximity structures [15-17].

\footnotetext{
*abay@chalmers.se

†per.delsing@chalmers.se
}

In spite of intensive research, no systematic investigation of the proximity effect in InAs nanowires has been reported, leaving open important questions about the consistency of the observed transport phenomena and theoretical views of the proximity effect.

In this paper, we report on extensive experimental studies of current-voltage characteristics (IVC) of a large variety of hybrid devices made with InAs nanowires connected to aluminum electrodes. These Al-InAs NW-Al devices include suspended and nonsuspended nanowires and nanowires with different lengths, which are tested at different temperatures, magnetic fields, and gate voltages. We measure the main proximity-effect characteristics: Josephson critical current, excess current, and subgap current features, and we make a quantitative comparison with relevant theoretical models.

Our main conclusion is that the most properties of the proximity effect can be qualitatively understood and quantitatively reasonably well fit on the basis of existing theory. In particular, the record high Josephson critical current of $800 \mathrm{nA}$, observed in the shortest studied nanowire (with $30 \mathrm{~nm}$ separation between superconducting electrodes) is close to the theoretical bound for ballistic point contacts [18]. In longer devices, the decay of the critical current with length is consistent with a crossover from the ballistic to diffusive transport regime, followed by a crossover from a short- to long-junction behavior.

In the gate-controlled suspended devices, we observe a crossover from a distinct S-normal-metal-S (SNS)-type behavior, with large positive excess current and enhanced subgap conductance, to tunneling S-insulator-S (SIS)-type behavior, in accordance with gradual depletion of the conducting channels by the gate potential and increase of the wire resistance. 
We also observe subgap current features associated with multiple Andreev reflection (MAR) transport [19]. In addition to those MAR features, we systematically observe subgap features, which are not associated with MAR but have some different origins. These features are not related to phononinduced resonances [20], and they do not seem to have an electromagnetic origin. They appear on the IVCs as voltage steps, strikingly similar to the voltage steps generated by phase slip centers in superconducting whiskers [21,22].

The structure of the paper is as follows: After describing the device fabrication and experimental setup in Sec. II, we summarize the normal-state conduction properties of the devices in Sec. III, which give the necessary input for choosing an appropriate theoretical model of the proximity effect described in Sec. IV. Then, in the following sections, we discuss the superconducting transport properties: excess current in Sec. V, Josephson current in Sec. VI, subgap current in Sec. VII, and variation of the current under the gate potential in Sec. VIII. Section IX contains conclusive remarks.

\section{EXPERIMENTAL DETAILS}

\section{A. Sample fabrication}

The devices we have investigated are of three types: nanowires placed directly on the substrate with either (a) two superconducting contacts or (b) multiple contacts, and (c) suspended devices with local gates (Fig. 1). All devices are made on standard $\mathrm{Si}$ substrates capped by 400 -nm-thick $\mathrm{SiO}_{2}$.

The nanowires are grown by chemical beam epitaxy [23]. In the growth process, metal-organic gaseous sources are thermally cracked to their components and the growth materials are directed as a beam towards an InAs substrate placed in the growth chamber. At the optimal temperature, the nanowire growth is catalyzed by Au aerosol particles that have been distributed on the substrate. The sizes of the $\mathrm{Au}$ seeds determine the diameter of the nanowires. In this paper, the nanowires are taken from a single growth batch with an average diameter of $80 \mathrm{~nm}$.

To fabricate the nonsuspended devices, InAs nanowires are first transferred to a Si substrate and their relative positions with respect to predefined marks are determined with the help of scanning electron microscope (SEM) images. The extracted locations are then used to pattern superconducting $\mathrm{Ti} / \mathrm{Al}$ (5/150-nm-thick) contacts on top of the nanowires. Depending on the intended device length, i.e., distance between source and drain electrodes, the superconducting contacts are defined by either single-step or double-step electron-beam (e-beam) lithography [11]. The shorter devices $(L<100 \mathrm{~nm})$ are defined by the double-step e-beam lithography, whereas the longer devices $(L \geqslant 100 \mathrm{~nm})$ are defined by the single-step e-beam lithography. A SEM image of a typical two-terminal device ( $L \approx 100 \mathrm{~nm}$ defined by the single e-beam lithography) is shown in Fig. 1(a). The inset image shows a short-length device of $L \approx 60 \mathrm{~nm}$ defined by the double-step e-beam lithography.

To fabricate the suspended devices, a standard Si substrate is first patterned with interdigitated Ti/Au stripes [24]. InAs nanowires are then transferred to the already patterned $\mathrm{Si}$ substrate and some of the nanowires end up on top of the (a)

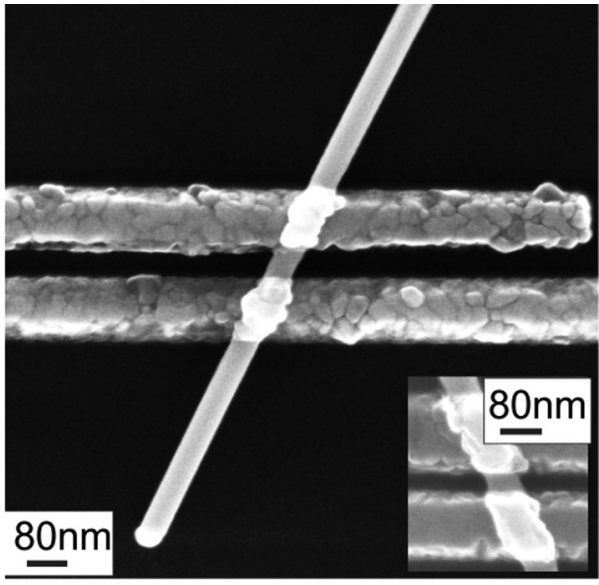

(b)

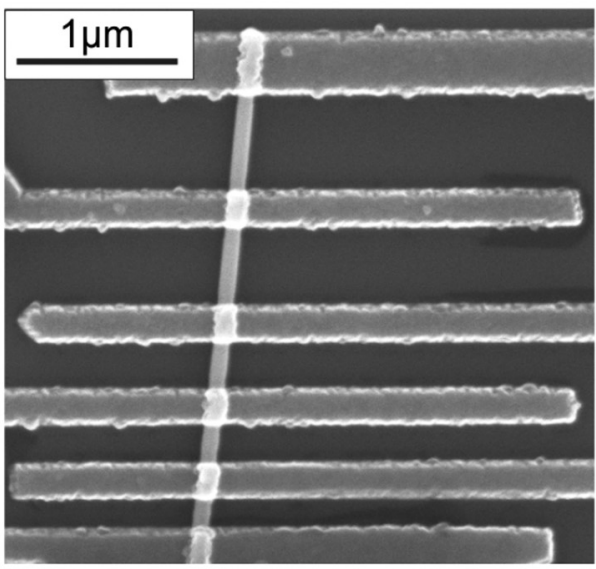

(c)

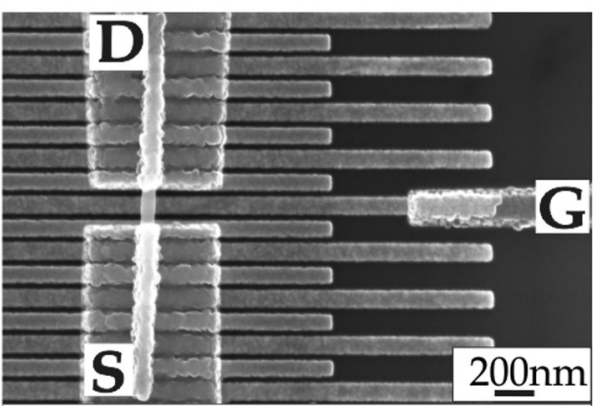

FIG. 1. SEM images of investigated devices. (a) Short-length device of type $A$ defined by a single-step electron-beam lithography (inset shows a short-length device defined by a double-step e-beam lithography). (b) Device of type $B$ has multiple contacts on a single nanowire separated by different lengths. (c) Three-terminal device with a suspended nanowire and a nearby local gate; the gate is $15 \mathrm{~nm}$ below the nanowire.

interdigitated metal stripes. The stripes are patterned in a two-step fabrication process in order to get a height difference of $15 \mathrm{~nm}$ between every two adjacent stripes. This allows the nanowires to rest on the thicker electrodes ( $65 \mathrm{~nm}$ thick), while being suspended above the substrate and the thinner electrodes (50 $\mathrm{nm}$ thick). With the help of SEM images, the positions of suitable nanowires are found and superconducting electrodes $\mathrm{Ti} / \mathrm{Al}(5 / 150 \mathrm{~nm}$ thick) are defined on selected nanowires with e-beam lithography. A SEM image of a suspended device is shown in Fig. 1(c). 


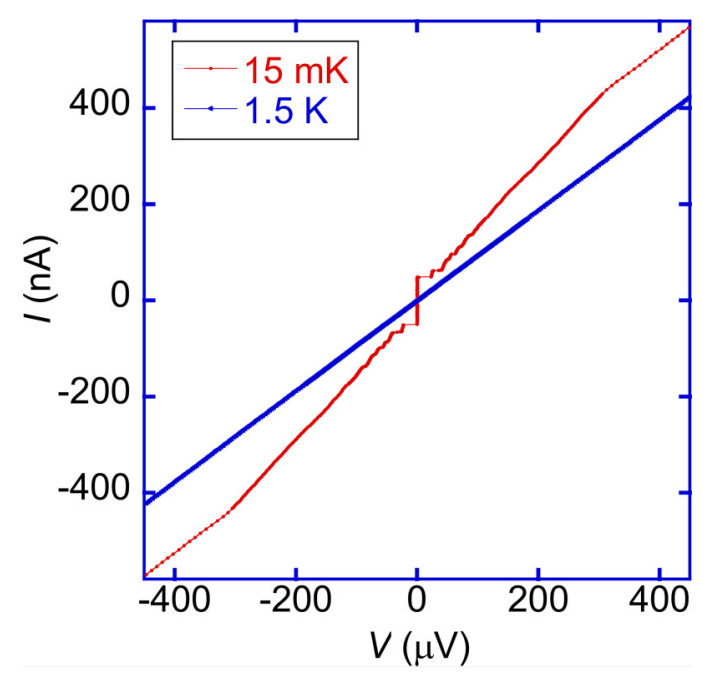

FIG. 2. (Color online) Normal-state IVC (blue line) and superconducting-state IVC (red line) for a device of length $L \approx$ $150 \mathrm{~nm}$ (sample $B_{5 a}$ ). The superconducting IVC shows normalstate ohmic behavior $\left(R_{n}=1.07 \mathrm{k} \Omega\right)$ at $|V|>2 \Delta / e$, enhanced conductance and current features at subgap voltages $|V| \leqslant 2 \Delta / e$, and the Josephson current.

To get good transparency of the metal-nanowire interfaces, an ammonium polysulfide solution $\left(\mathrm{NH}_{4} \mathrm{~S}_{x}\right)$ cleaning process $[11,25]$ has been used prior to evaporation of the superconducting contacts. The samples are then characterized at room temperature and stored in a vacuum box before further measurements at low temperatures.

\section{B. Experimental setup}

Current-voltage characteristics of the devices are measured in a dilution refrigerator with a base temperature of $15 \mathrm{mK}$. The IVCs are recorded in either a current- or voltage-bias configuration. In the current-bias mode, the current is determined by a high-resistance bias resistor in series with the device. As we increase the current, the voltage across the device is simultaneously measured with a differential amplifier. In the voltage-bias mode, a voltage is directly applied across the device while the current is measured simultaneously by a transimpedance amplifier. To decrease noise coupling to the devices, the electrical lines in the measurement setup are well filtered and thermally anchored at different temperature stages of the refrigerator. The measurement setup is also designed to measure IVCs as a function of temperature and magnetic field.

\section{Current-voltage characteristics}

A typical IVC is shown in Fig. 2 for a device of length $L \approx 150 \mathrm{~nm}$ (sample $B_{5 a}$ in Table I). Above the critical temperature, the IVC (blue line) exhibits ohmic behavior with a normal-state resistance of $R_{n}=1.07 \mathrm{k} \Omega$. The critical temperature, $T_{c}=1.1 \mathrm{~K}$, for the devices was determined from samples with shorted electrodes, i.e., without any nanowire. This value agrees well with the temperature at which the Josephson current disappears in the samples with strong Josephson coupling. At temperatures well below $T_{c}$, the IVC (red line) shows three distinct conductance regimes: (i) For
TABLE I. Measurement values for devices of different types. Type- $A$ devices are nanowires with two superconducting contacts. Type- $B$ devices are defined on a single nanowire with multiple contacts. Adjacent junctions in the $B$-type devices are marked with alphabetic letters. Type- $C$ devices are suspended devices with local gates. The nanowires are taken from the same growth batch of approximately $80 \mathrm{~nm}$ in diameter.

\begin{tabular}{lcccrcc}
\hline \hline Device & & $L(\mathrm{~nm})$ & $R_{n}(\mathrm{k} \Omega)$ & $I_{m}(\mathrm{nA})$ & $e I_{m} R_{n} / \Delta$ & $e I_{\mathrm{exc}} R_{n} / \Delta$ \\
\hline$A$ & 1 & 30 & 0.16 & 800 & 1.02 & 1.52 \\
& 2 & 90 & 0.55 & 95 & 0.40 & 0.87 \\
& 3 & 100 & 0.56 & 54 & 0.23 & 0.75 \\
& 4 & 220 & 1.04 & 30 & 0.24 & 0.76 \\
$B$ & $5 a$ & 150 & 1.07 & 50 & 0.41 & 1.20 \\
& $5 b$ & 170 & 1.28 & 40 & 0.39 & 1.28 \\
& $5 c$ & 180 & 1.34 & 36 & 0.37 & 1.31 \\
& $5 d$ & 190 & 1.37 & 35 & 0.36 & 1.11 \\
& $6 a$ & 110 & 1.84 & 23 & 0.32 & 1.21 \\
& $6 b$ & 200 & 2.40 & 12 & 0.21 & 0.81 \\
& $6 c$ & 250 & 2.72 & 9 & 0.20 & 0.77 \\
& $6 d$ & 500 & 4.21 & 3 & 0.10 & 0.71 \\
& $6 e$ & 600 & 4.82 & 1 & 0.04 & 0.64 \\
$C$ & 7 & 200 & 2.23 & 15 & 0.24 & 1.30 \\
& 8 & 150 & 3.3 & 13 & 0.34 & 1.17 \\
& 9 & 130 & 2.19 & 28 & 0.47 & 1.02 \\
& 10 & 300 & 3.80 & 6 & 0.17 & 1.23 \\
& 11 & 150 & 5.01 & 2.6 & 0.10 & 0.78 \\
& 12 & 200 & 3.6 & 7.5 & 0.21 & 1.11 \\
\hline \hline
\end{tabular}

voltages $|V|>2 \Delta / e$, the IVC shows a linear behavior with the same resistance as in the normal state, $R_{n}$. (ii) For smaller voltages $|V|<2 \Delta / e$, the resistance is approximately $R_{n} / 2$ and exhibits subgap features. (iii) At the zero voltage $V=0$, the device switches to zero resistance, exhibiting a Josephson current.

In the next sections, we perform quantitative analysis of the IVC based on a detailed characterization of the normal-state current transport in the wire.

\section{NORMAL-STATE TRANSPORT}

In order to characterize the normal-state properties of the junctions, dc measurements have been performed on several devices with a broad range of lengths and resistances. Measurement results for representative devices are summarized in Table I. The devices are divided into three groups, $A, B$, and $C$, corresponding to the two-terminal, multiterminal, and suspended devices, respectively, as shown in Fig. 1.

The normal-state resistance as a function of length for devices $B_{5}$ and $B_{6}$ is plotted in Fig. 3. The resistance of each device increases linearly with length, with approximately the same resistance per unit length, $R / L \approx 6 \Omega / \mathrm{nm}$. Here, the resistance values are taken from the two-point measurements that also include interface resistance. From the length dependence of the resistance in Fig. 3, we extract the contact resistance by extrapolating to zero length. For device $B_{5}$, we find that contact contribution is less than $180 \Omega$, while for device $B_{6}$, it is approximately $1.2 \mathrm{k} \Omega$. 


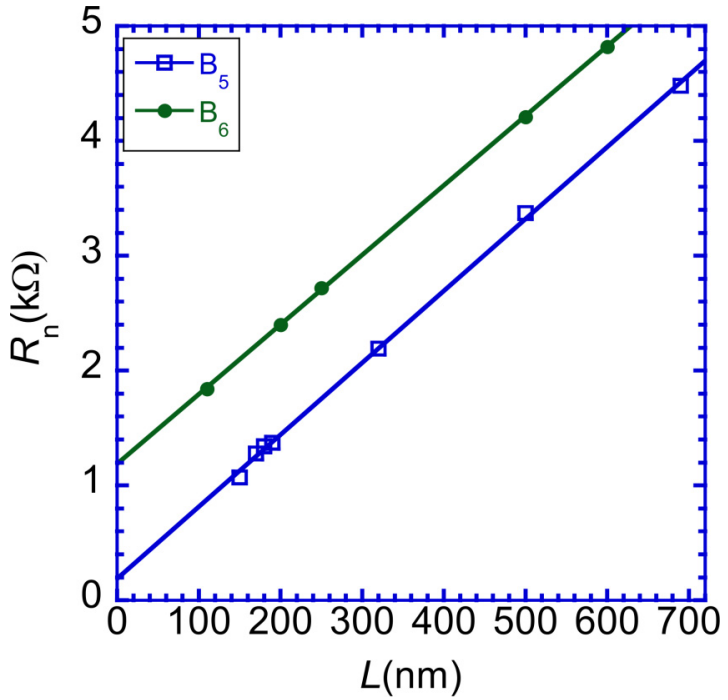

FIG. 3. (Color online) Normal-state differential resistance as a function of length for devices $B_{5}$ (blue line) and $B_{6}$ (green line). The two devices show approximately the same resistance per unit length $6 \Omega / \mathrm{nm}$. Extrapolation to zero length gives the full contact resistance, which is less than $180 \Omega$ for $B_{5}$ and $1.2 \mathrm{k} \Omega$ for $B_{6}$.

Taking advantage of multiple contacts of the $B$-type devices, we perform two- and four-point measurements of the resistance, which allows us to determine the number of conducting channels and the channel average transparency. The two- and four-point resistance expressions can be written for perfect interfaces as $R_{2 p}=R_{q} /\left(N T_{t}\right)$ and $R_{4 p}=R_{q}(1-$ $\left.T_{t}\right) /\left(N T_{t}\right)$, respectively [26], where $R_{q}=h / 2 e^{2}=12.9 \mathrm{k} \Omega$ is the quantum of resistance, $N$ is the number of channels, and $T_{t}$ is the average transparency of the channels. Taking two- and four-point resistance measurements on the same section, we find that our nanowires have a spread in the number of channels ranging between 50 and 100 channels. For instance, for junction $B_{5 c}$, we measured $R_{2 p}=1.34 \mathrm{k} \Omega$ and $R_{4 p}=1.18 \mathrm{k} \Omega$, giving the number of channels,

$$
N=\frac{R_{q}}{R_{2 p}-R_{4 p}} \approx 80 .
$$

This is consistent with the contact resistance found for device $B_{5}$, and implies perfect S-NW interfaces with transparency close to unity. For the same junction, we can then extract the average transparency $T_{t}=0.12$ for the channels.

Assuming only a surface layer of nanowire to be conducting, in analogy with the 2-Dimensional Electron Gas (2DEG) conductivity in planar InAs devices, such a large amount of conducting channels would give an unrealistically small value for the Fermi wavelength. On the other hand, assuming the whole bulk of the wire to be conducting, we find the electronic Fermi wavelength $\lambda_{F}$ to depend logarithmically on the number of channels, as

$$
\lambda_{F}(N)=\frac{2 \pi r_{w}}{\alpha_{l, n}} \approx A-B \ln (N) .
$$

This result is arrived at by solving the Schrödinger equation in a cylinder of radius $r_{w}$ and counting the number of modes (channels) that cross the Fermi level. In Eq. (2), $\alpha_{l, n}$ is the
TABLE II. Summary of the extracted parameters for the nanowires.

\begin{tabular}{lc}
\hline \hline Fermi wavelength $\lambda_{F}$ & $22 \mathrm{~nm}$ \\
Fermi wave vector $k_{F}$ & $2.9 \times 10^{8} \mathrm{~m}^{-1}$ \\
Fermi velocity $v_{F}$ & $1.3 \times 10^{6} \mathrm{~m} / \mathrm{s}$ \\
Mean free path $\ell_{e}$ & $46 \mathrm{~nm}$ \\
No. conducting channels & 55 \\
Superconducting gap $\Delta$ & $160 \mu \mathrm{eV}$ \\
Clean coherence length $\xi_{0}$ & $1300 \mathrm{~nm}$ \\
Diffusive coherence length $\xi_{D}$ & $245 \mathrm{~nm}$ \\
Diffusion constant $D_{\text {diff }}$ & $200 \mathrm{~cm}^{2} / \mathrm{s}$ \\
$e \Delta / \pi \hbar$ & $12.6 \mathrm{nA}$ \\
\hline \hline
\end{tabular}

$n$th zero of the $J_{l}$ th Bessel function, where $l$ labels the last mode that contributes to transport. The coefficients $A$ and $B$ in Eq. (2) are functions of the nanowire radius; for $r_{w}=40 \mathrm{~nm}$, $A=42.4 \mathrm{~nm}$ and $B=7.63 \mathrm{~nm}$, and varying the radius by $\pm 10 \%$ changes both coefficients by approximately $\pm 2 \%$. We can bracket the Fermi wavelength between $\lambda_{F} \approx 17 \mathrm{~nm}$ (100 channels) and $\lambda_{F} \approx 22 \mathrm{~nm}$ (50 channels) for $r_{w}=$ $40 \mathrm{~nm}$. Our values are consistent with the ones reported for planar InAs $2 \mathrm{DEG}\left(\lambda_{F} \approx 18 \mathrm{~nm}\right)$ [27] and for InAs nanowires (22 $\lesssim \lambda_{F} \lesssim 33 \mathrm{~nm}$ ) [7]. In the further discussions, we adopt the values $\lambda_{F}=22 \mathrm{~nm}$ and $N=55$ for all of the junctions.

Furthermore, we use the measured resistance per unit length, $R / L=6 \Omega / \mathrm{nm}$, together with the expression for the Drude conductivity, $\sigma=n e^{2} \tau / m$, to evaluate a mean free path for the nanowires of $\ell_{e}=46 \mathrm{~nm}$. The corresponding Fermi velocity $v_{F}=\hbar k_{F} / m^{*} \approx 1.3 \times 10^{6} \mathrm{~m} / \mathrm{s}$ is evaluated by using an electronic effective mass $m^{*}=0.026 m_{e}$ of bulk InAs, where $m_{e}$ is the free-electron mass. The effective mass of electrons $m^{*}$ for planar InAs 2DEG has been estimated to be in a range from 0.024 to $0.04 m_{e}[27,28]$. The normal-state properties of the nanowires are summarized in Table II.

\section{THEORETICAL MODEL}

The major difficulty for theoretical interpretation of the experimental data is the very large spread of the wire lengths. Indeed, the shortest wire $(30 \mathrm{~nm})$ is in the ballistic point contact regime $\left(L<\ell_{e}, \xi_{0}\right.$, with $\left.\xi_{0}=\hbar v_{f} / 2 \pi k_{B} T_{c}\right)$, while the longest wire is in the diffusive long-junction regime $\left(L>\ell_{e}, \xi_{D}\right.$, with $\left.\xi_{D}=\sqrt{\ell_{e} \xi_{0}}\right)$. The majority of the junctions are in the intermediate crossover region. Furthermore, all of the tested junctions exhibit the Josephson effect. This implies that the current transport is fully coherent and requires theoretical modeling within the framework of the coherent MAR theory [29-32]. To overcome this difficulty, we adopt a simple and tractable model, with which we can bridge between the ballistic and diffusive transport regimes, and describe the crossover to the long-junction behavior [33]. The model setup is shown in Fig. 4. We assume that the two superconducting leads are connected to the nanowire by highly transmissive contacts, which are treated as fully transparent. The nanowire is disordered due to elastic scattering by impurities and crystal imperfections. This is treated in the Born approximation, and the mean free path estimated from the experiments $\ell_{e}=46 \mathrm{~nm}$ infers a scattering rate $\Gamma=v_{F} / \ell_{e} \approx 2.8 \times 10^{13} \mathrm{~s}^{-1}$. Strong defects are included and treated as a single interface having 


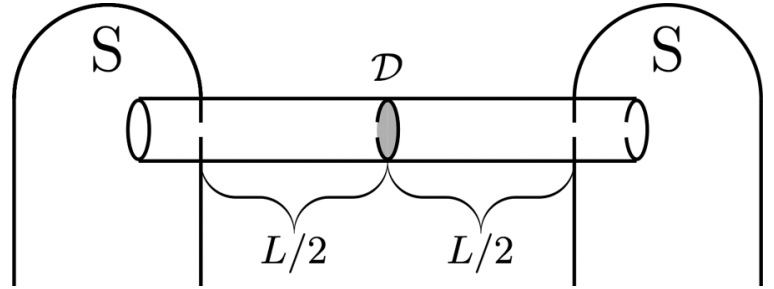

FIG. 4. A schematic picture of the theoretical model for the nanowire junctions: Superconducting electrodes (S) are connected by a disordered nanowire of length $L$, which also contains crystalline defects. The latter are modeled with a lumped scatterer situated in the middle of the wire and having effective transparency $\mathcal{D}$. The applied voltage is assumed to mostly drop at the scatterer.

the same effective transparency $\mathcal{D}$ for all conducting channels. This defect is assumed to be in the center of the nanowire, and the applied voltage is assumed to drop at the defect.

Using the quasiclassical Green's function methods described in Refs. [34,35], we calculate the IVC as a function of device length and transparency by solving the coherent MAR problem. The current is calculated at the scatterer [34] and expressed through the boundary values, $\hat{g}_{R / L}=\hat{g}\left(\hat{\vec{p}}_{F}, x=\right.$ $\pm 0 ; \omega)$, of the quasiclassical Green's function for a given channel,

$\hat{g}\left(\hat{\vec{p}}_{F}, x ; \omega\right)=\left(\begin{array}{cc}g\left(\hat{\vec{p}}_{F}, x ; \omega\right) & f\left(\hat{\vec{p}}_{F}, x ; \omega\right) \\ \tilde{f}\left(\hat{\vec{p}}_{F}, x ; \omega\right) & -g\left(\hat{\vec{p}}_{F}, x ; \omega\right)\end{array}\right), \quad \hat{g}^{2}=-\pi^{2}$.

The Green's function is computed by solving the Eilenberger equation in the right and left parts of the nanowire,

$$
\begin{aligned}
& i \hbar \vec{v}_{F} \cdot \vec{\partial}_{x} \hat{g}\left(\hat{\vec{p}}_{F}, x ; \omega\right) \\
& \quad+\left[\varepsilon(x ; \omega) \hat{\tau}_{3}-\hat{\Delta}_{\text {imp }}(x ; \omega), \hat{g}\left(\hat{\vec{p}}_{F}, x ; \omega\right)\right]=0,
\end{aligned}
$$

complemented with the Zaitsev boundary conditions at the scatterer and NW-S interfaces [34-36]. $\hat{\tau}_{3}$ is the third Pauli matrix in Nambu space. In Eq. (4), we introduce the impurity scattering via the impurity self-energies,

$$
\begin{aligned}
\varepsilon(x ; \omega) & =\hbar \omega-\hbar \Gamma\left\langle g\left(\hat{\vec{p}}_{F}, x ; \omega\right)\right\rangle_{p_{F}}, \\
\hat{\Delta}_{\text {imp }}(x ; \omega) & =\hbar \Gamma\left\langle\hat{f}\left(\hat{\vec{p}}_{F}, x ; \omega\right)\right\rangle_{p_{F}},
\end{aligned}
$$

where $\langle\cdot\rangle_{p_{F}}$ is average over directions $\left( \pm \hat{\vec{p}}_{F}\right)$. The matrix $\hat{f}$ is the anomalous (off-diagonal) part of the Green's function $\hat{g}$. The components $(f, \tilde{f})$ of $\hat{f}$ describe the pairing correlations leaking into the nanowire and the two are related by symmetry as $\tilde{f}\left(\hat{\vec{p}}_{F}, x ; \omega\right)=-f^{*}\left(-\hat{\vec{p}}_{F}, x ;-\omega^{*}\right)$.

\section{EXCESS CURRENT}

We start with a discussion of the excess current at large voltage, which is a robust feature of the proximity IVC. The excess current $I_{\mathrm{exc}}$ is extracted from the current-voltage characteristics at large voltage bias using the asymptotic form $I(V>2 \Delta / e) \approx V / R_{N}+I_{\mathrm{exc}}+O(\Delta / \mathrm{eV})$. The excess current contains contributions both from the single-particle and from the two-particle Andreev currents, and it linearly scales with the energy gap $\Delta(T)$ (see, e.g., Ref. [37]). In Fig. 5(a), the
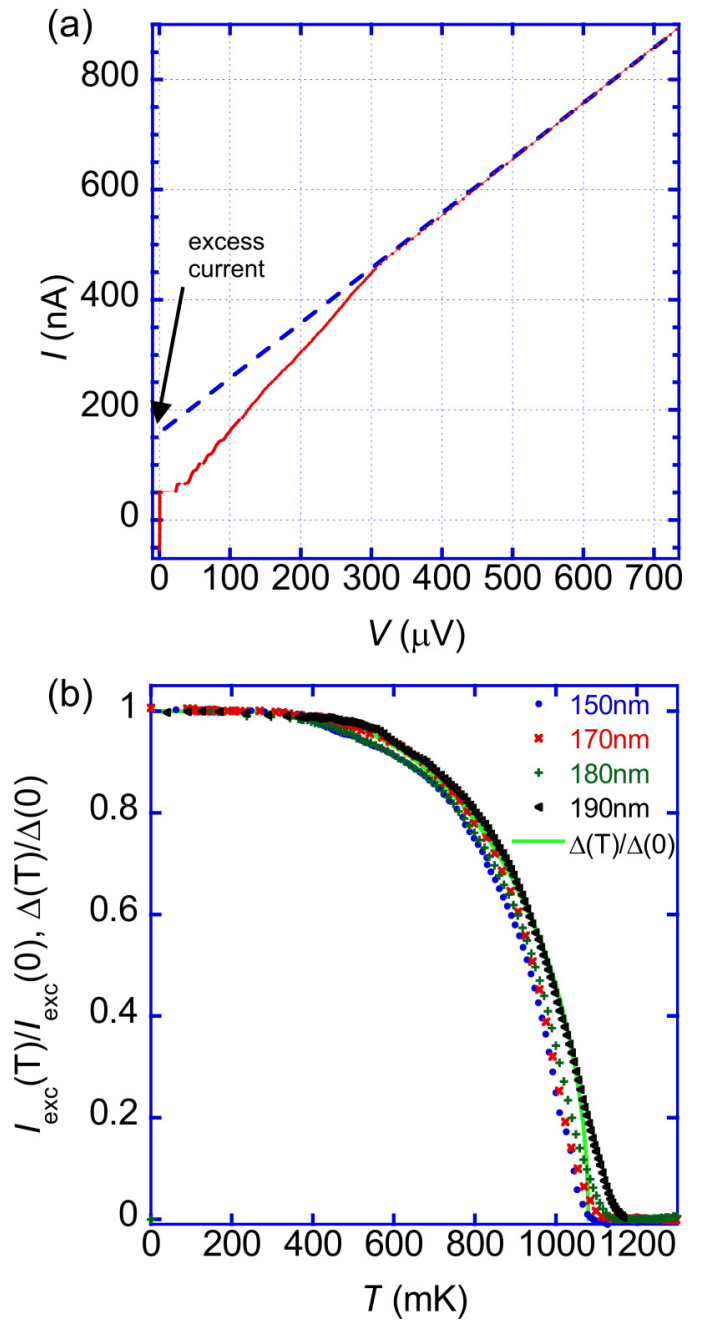

FIG. 5. (Color online) (a) Current-voltage characteristics of the device $B_{5 a}$ with length $L=150 \mathrm{~nm}$ and normal-state resistance $R_{n}=$ $1.07 \mathrm{k} \Omega$. The excess current is extracted by extrapolating the IVC from high voltage to zero voltage. (b) Excess currents as a function of temperature are shown for device $B_{5}(L=150,170,180$, and $190 \mathrm{~nm})$. The excess currents follow the superconducting energy gap $\Delta(T)$ (light green line).

excess current is obtained for the device $B_{5 a}$ by extrapolating a linear fit of the IVC measured at $V>2 \Delta / e$ (blue dashed line) giving $I_{\mathrm{exc}}=150 \mathrm{nA}$. To verify that the measured excess current derives from Andreev scattering processes, the experimentally extracted excess current is plotted as a function of temperature in Fig. 5(b). As can be seen, the amplitude of the excess current follows the temperature dependence of the superconducting gap $\Delta(T)$.

The excess current also depends on the transparency and the length of the nanowire device. In Fig. 6, we present the computed excess current as a function of device length together with $I_{\text {exc }}$ extracted from the measurements. The maximum values of the theoretical curves correspond to the point-contact limit $(L=0)$, and they are in good agreement with analytical results [37], $I_{\mathrm{exc}}=(8 / 3 \pi)(e \Delta / \hbar)$ per channel for $\mathcal{D}=1$ and $I_{\mathrm{exc}} \approx \mathcal{D}^{2}(e \Delta / \hbar)$ for $\mathcal{D} \ll 1$. When the wire length is increased, the excess current decreases. This was 


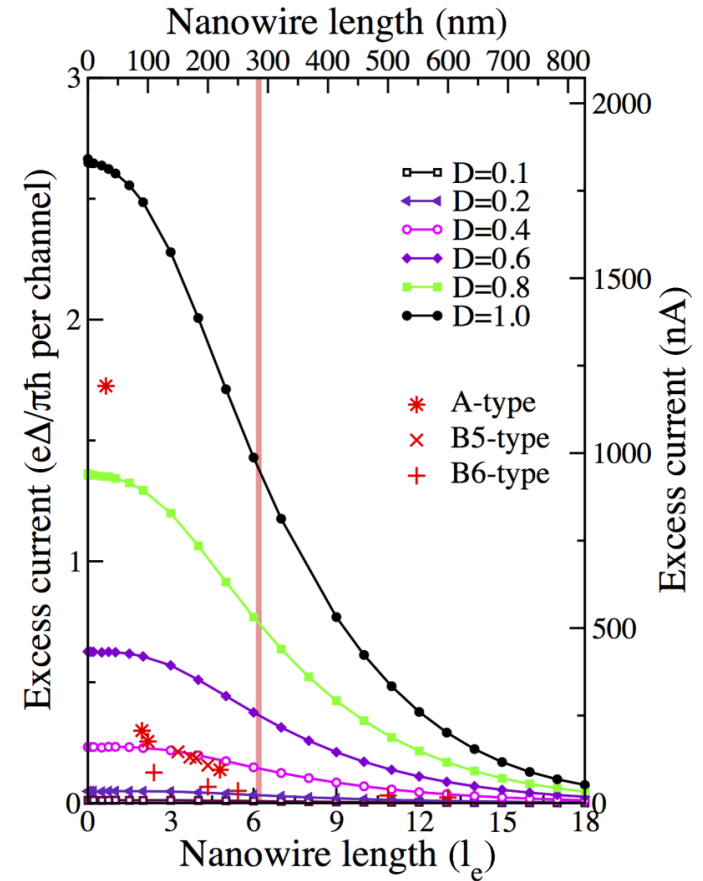

FIG. 6. (Color online) The computed excess current as a function of length and effective transparency of the device. The units of the excess current are given on the left axis for the single channel and on the right in $\mathrm{nA}$ assuming that all channels have the same average transparency. The length of the device is given both in units of the mean free path (bottom $x$ axis) and in nm (top $x$ axis). The vertical line indicates the length $L=\sqrt{\hbar D_{\text {diff }} / \Delta} \approx 1.1 \xi_{D}$, where the Thouless energy, $E_{\mathrm{Th}}=\hbar D_{\text {diff }} / L^{2}$, equals the superconducting gap; this length separates the short-junction limit $\left(E_{\mathrm{Th}} \gg \Delta\right)$ from the long-junction limit $\left(E_{\mathrm{Th}} \ll \Delta\right)$. The stars and crosses are the experimental data from Table I; as can be seen, the most devices are in the intermediate limit where $E_{\mathrm{Th}} \approx \Delta$.

also found, experimentally and theoretically, in ballistic 2DEG InAs [27], and computed for fully diffusive junctions [38,39]. In our case, the experimental values fall on curves with a typical effective transparency between 0.2 and 0.4 being only weakly device dependent between batches of nanowires. These values compare favorably with $T_{t}=0.12$ extracted from the two-point and four-point measurements in the normal state.

One device, $A_{1}(L=30 \mathrm{~nm})$, however, stands out, showing a high transparency of $\mathcal{D} \approx 0.87$. For this junction, with highly transmissive ballistic point contact, one should anticipate the largest critical current.

\section{JOSEPHSON CURRENT}

Next, we discuss the Josephson critical current as a function of length, temperature, and magnetic field. The maximum values of the Josephson current, $I_{m}$, are extracted from the experimentally obtained IVC at the base temperature of $15 \mathrm{mK}$ and are shown in Table I. The maximum currents exhibit a range of values depending on the resistance and length of the devices, from a few to $800 \mathrm{nA}$. Similarly, the characteristic voltage, i.e., the $I_{m} R_{n}$ product, also exhibits a range of values, from 20 to $130 \mu \mathrm{V}$.

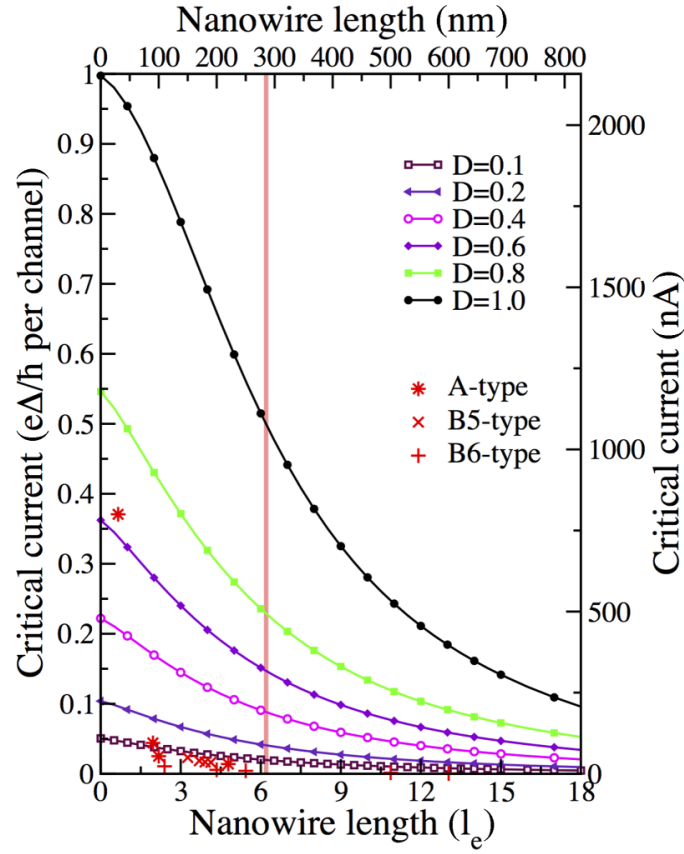

FIG. 7. (Color online) The computed critical current $I_{c}$ as a function of length and effective transparency of the device. The units of the maximum current are given on the left axis for the single channel and on the right in nA assuming that all channels have the same average transparency. The length of the device is given both in units of the mean free path (bottom $x$ axis) and in nm (top $x$ axis). The single markers are the experimental values of the maximum Josephson current $I_{m}$ reported in Table I.

Theoretically, the Josephson current-phase relation is computed using boundary values of the Green's function, $\hat{g}\left(\hat{\vec{p}}_{F}, x ; \omega\right)$, in Eqs. (3) and (4). The expression reads [36]

$$
I_{s}(\phi)=\frac{8 \pi e T \mathcal{D}}{h} \sum_{\omega_{n}>0}\left\langle\frac{f_{R} f_{L} \sin \phi}{2-\mathcal{D}\left(g_{R} g_{L}-f_{R} f_{L} \cos \phi+1\right)}\right\rangle_{p_{F}} .
$$

The sum is over all Matsubara frequencies, $\omega_{n}=\pi k_{B} T(2 n+$ 1), $T$ is the temperature, and $\phi$ is the phase difference over the junction. The critical current is obtained by maximizing the supercurrent.

The maximum Josephson currents presented in Table I, together with a theoretical critical current fit, as a function of length, are plotted in Fig. 7. The shortest junction exhibits the largest Josephson current, as expected, with the theoretical fit of the transparency, $\mathcal{D} \approx 0.65$. This is very close $(75 \%)$ to the theoretical limit defined by the transparency extracted from the analysis of the excess current. The other junctions fall in the transparency region $0.05<\mathcal{D}<0.1$, which is smaller (approximately by a factor of 4 ) compared to the transparency extracted from the excess current.

A similar or even larger reduction of Josephson current is commonly observed in nanowires, and it is also common in 2DEG InAs Josephson junctions [27]. Such an effect is not well understood; perhaps it could be related to some depairing mechanism, for example, due to magnetic scattering.

One would expect a certain suppression of the Josephson current extracted from the IVC measurement compared to 


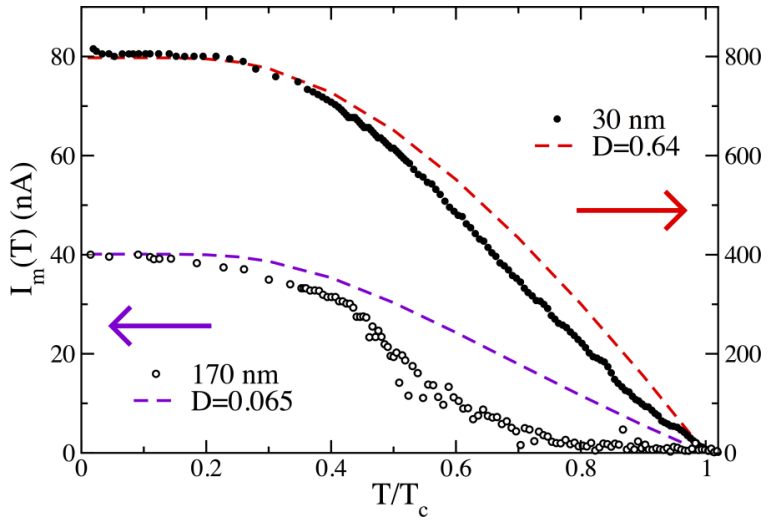

FIG. 8. (Color online) Maximum Josephson current as a function of temperature for two devices of length $L=30$ and $L=170 \mathrm{~nm}$. Note the different scales for the current magnitude. Theoretical fits to the critical current for both devices are shown. The transparency chosen to fit the data is taken from the low-temperature values for $I_{m}$ in Fig. 7.

the equilibrium critical current due to the effect of phase fluctuations. However, our analysis shows that the majority of our junctions are overdamped, and the suppression of the critical current in this regime is relatively small and cannot account for the whole suppression effect. Indeed, the capacitances of the devices are estimated in the range $C \sim 1-5 \mathrm{fF}$; cf. Ref. [11]. Assuming $C=5 \mathrm{fF}$ and the junction resistance $R_{0} \sim 100 \Omega$ at plasma frequency $\sim 1 \mathrm{GHz}$ corresponding to the free-space impedance, we estimate the quality factor $Q=\sqrt{2 e I_{c} C / \hbar} R_{0} \lesssim 0.1$ for the representative junction with critical current, $I_{m}=50 \mathrm{nA}$. This estimate refers to an unbiased junction; the $Q$ factor further decreases when the current bias is applied. For such an overdamped regime, $Q \ll 1$, the switching probability is significantly suppressed [40] and IVC can be modeled with the Ambegaokar-Halperin theory [41]. This conclusion is supported by the absence of hysteresis on IVC. The IVC measurement takes approximately one minute, so that the sample spends approximately a few seconds close to $I_{m}$. Assuming the temperature of electromagnetic fluctuations is close to the base temperature of $15 \mathrm{mK}$ due to a careful noise filtering [42], we find that the suppression effect accounts for approximately $20 \%$ of the theoretical value for the majority of the junctions with critical currents exceeding $10 \mathrm{nA}$. For the shortest junction with $I_{m}=800 \mathrm{nA}$, the suppression is even smaller: about a few percent.

The maximum Josephson current is also investigated as a function of temperature for several devices. The maximum currents for the shortest-length device $A_{1}(L \approx 30 \mathrm{~nm}$ and $R_{n}=0.16 \mathrm{k} \Omega$ ) and for the somewhat longer device $B_{5 b}$ ( $L \approx 170 \mathrm{~nm}$ and $R_{n}=1.15 \mathrm{k} \Omega$ ) are shown in Fig. 8. At the base temperature $T=15 \mathrm{mK}$, the devices have maximum Josephson currents of $I_{m}=800$ and $40 \mathrm{nA}$, respectively. The data for the shortest device agree well with theory in a broad range of temperatures. The longer device exhibits a concave-shaped decay at higher temperatures and deviates from the theoretical fit. The qualitatively similar shape of $I_{c}(T)$ has been theoretically found for diffusive junctions with highly resistive interfaces (SINIS) [43] and explained with enhancement of electron-hole dephasing in the proximity
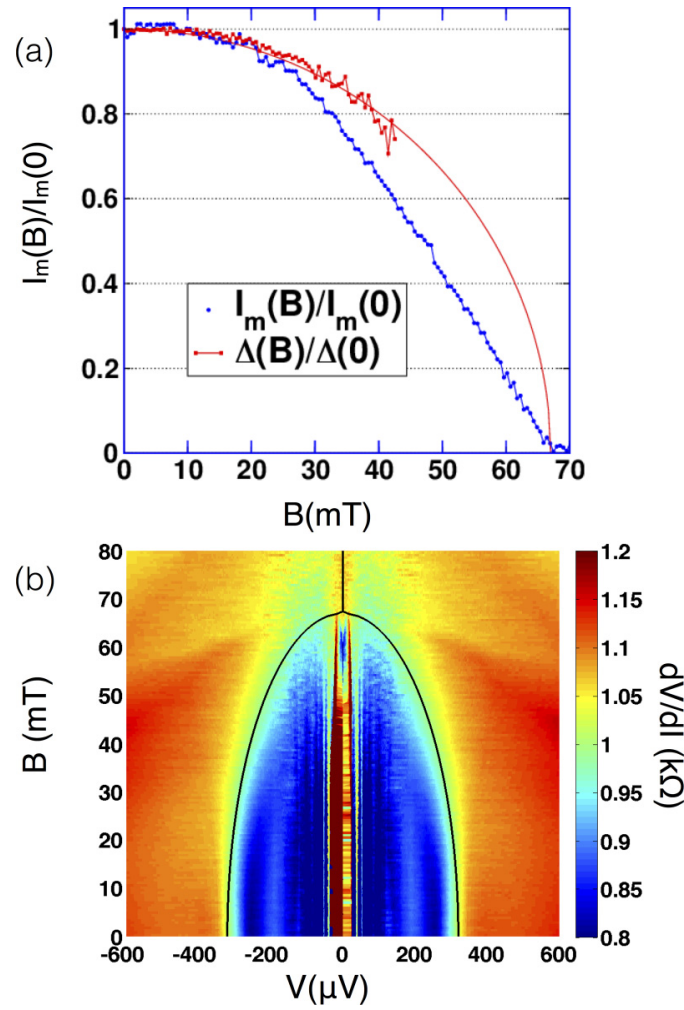

FIG. 9. (Color online) (a) Normalized maximum Josephson current (blue dots) as a function of magnetic field for device $B_{5 b}$ $(L=170 \mathrm{~nm})$ along with the extracted superconducting energy gap (red dots). The red line is $\Delta(B)=\Delta(0) \sqrt{1-\left(B / B_{c}\right)^{2}}$. This fit (black line $)$ is made to the gaplike feature in the $d V / d I(V, B)$ data displayed in (b).

region due to large dwell time. Such an effect is similar to the effect of increasing length of the junction (cf. Ref. [39]). Given such a similarity, we may conclude that although device $B_{5}$ has transparent S-NW interfaces, the model [43] might better capture the effect of the junction length.

At the base temperature of $15 \mathrm{mK}$, we also have obtained IVCs as a function of magnetic field. The magnetic field is applied perpendicular to the superconducting leads. The normalized maximum Josephson current and the superconducting energy gap as a function of magnetic field are plotted in Fig. 9 for device $B_{5 b}$ with $L=170 \mathrm{~nm}$. The superconducting gap $\Delta(B)$ is fitted to the expression $\Delta(B)=\Delta(0) \sqrt{1-\left(B / B_{c}\right)^{2}}$ from which we extract $B_{c}=67 \mathrm{mT}$. The maximum current decreases and is totally suppressed above $B_{c}$. No Fraunhofer oscillations are observed in any of the devices, consistent with a suppression of superconducting energy gap in the leads.

\section{SUBGAP CURRENT}

Now we proceed with discussion of the IVC in the subgap region, $V<2 \Delta / e$, as a function of temperature and magnetic field, and for different nanowire lengths. A typical plot of the differential resistance as a function of voltage is presented in Fig. 10(a). The resistance drops from $R_{n}=1.15 \mathrm{k} \Omega$ at $V \gg 2 \Delta$ to $R_{\mathrm{SG}} \approx 0.7 \mathrm{k} \Omega$ at $V \approx 260 \mu \mathrm{V}$, which corresponds to the gap value, $2 \Delta / e$. Such a drop of resistance in the subgap 

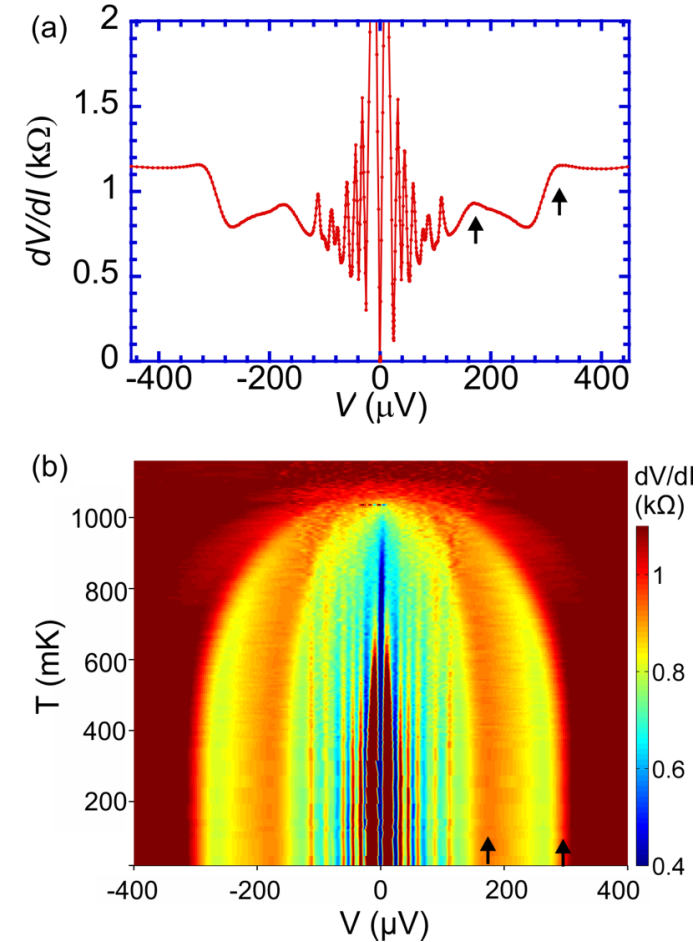

FIG. 10. (Color online) (a) Differential resistance $d V / d I$ as a function of voltage for device $B_{5 b}\left(L=170 \mathrm{~nm}\right.$ and $\left.R_{n}=1.15 \mathrm{k} \Omega\right)$. The resistance substantially decreases at $V \approx 2 \Delta / e$ and exhibits symmetric resistance (peaks/dips). (b) An image plot of differential resistance $d V / d I$ as a function of voltage and temperature. As the temperature is increased, the first two peaks, marked by two arrows, smoothly move towards lower voltages, consistent with the decrease of the superconducting energy gap $\Delta(T)$. However, the voltage positions of the other peaks are independent of temperature.

region is a characteristic of Andreev transport in transmissive SNS junctions [44]. Furthermore, the differential resistance shows a second feature at approximately half the gap voltage, $V \approx 130 \mu \mathrm{V}=2 \Delta / 2 e$ [shown by the arrow in Fig. 10(a)].

The positions of both of these features scale with the temperature dependence of the superconducting gap $\Delta(T)$, as shown in Fig. 10(b). This unambiguously indicates the MAR transport mechanism. Similar features associated with MAR are observed in all measured devices; in some devices, we also observed a third MAR feature at $2 \Delta / 3 e$.

We have also measured the dependence of positions of the resistance features as a function of magnetic field. The differential resistance of device $A_{4}$ as a function of magnetic field is shown in Fig. 11. In this device, the three MAR features are present (marked by arrows), which move smoothly towards lower voltages following the magnetic field dependence of the gap $\Delta(B)$.

Besides the MAR features, the IVC of all the measured devices exhibit a number of structures at lower voltages, whose positions are independent of both temperature and magnetic field; see Figs. 10 and 11. These structures are therefore not associated with MAR. However, they are related to the superconducting state in the electrodes since they do not persist above the critical temperature and critical magnetic field, and even disappear somewhat earlier.
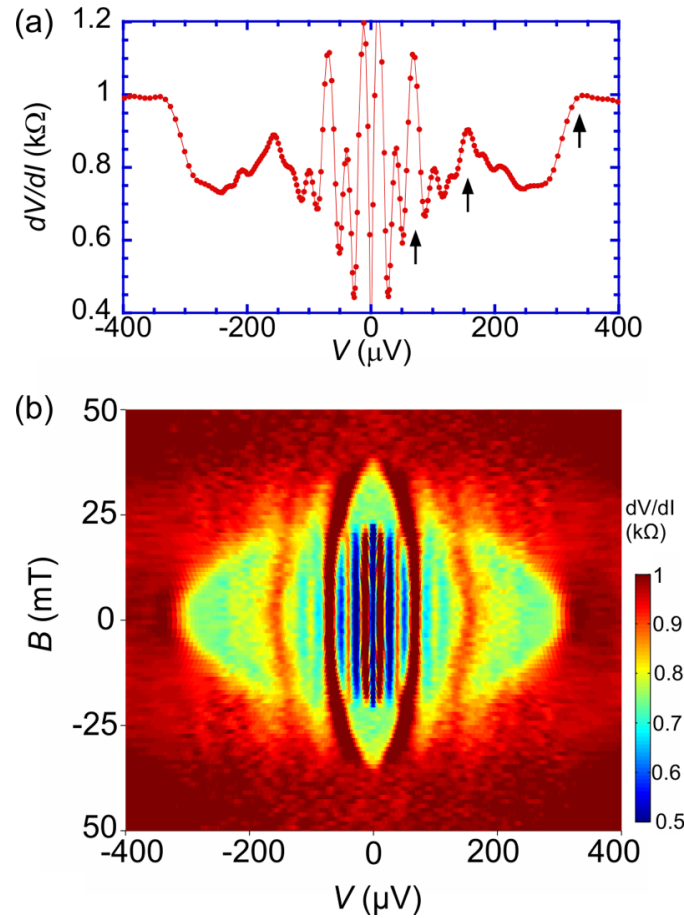

FIG. 11. (Color online) (a) Differential resistance $d V / d I$ as a function of voltage for device $A_{4}$. There are three MAR features indicated with arrows. (b) An image plot of differential resistance $d V / d I$ as a function of voltage and magnetic field. As the magnetic field is increased, the MAR resistance peaks smoothly move toward lower voltages, consistent with the decrease of the superconducting energy gap $\Delta(B)$. However, the voltage positions of the other peaks are independent of magnetic field.

The origin of these structures is not clear. In Ref. [20], similar structures were reported for suspended NW devices and attributed to resonances resulting from coupling of the ac Josephson current to mechanical vibrations in the wire. The

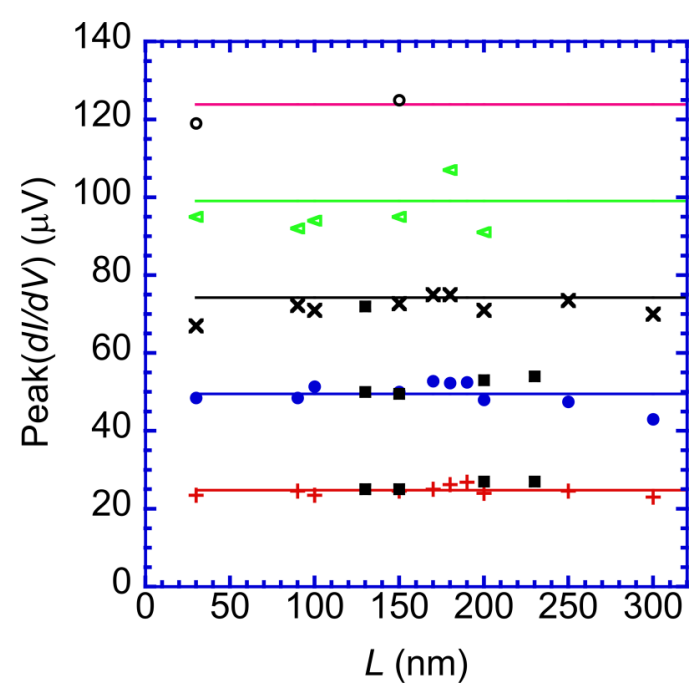

FIG. 12. (Color online) Voltage positions of the temperatureindependent differential conductance peaks as a function of length for devices of type $A, B$, and $C$. Horizontal lines indicate the multiples of the voltage $V \approx 24 \mu \mathrm{V}$. 
fact that we observe such structures not only in suspended but also in nonsuspended wires rules out this explanation. Furthermore, the phonon resonances would appear at voltages corresponding to the phonon eigenfrequencies, i.e., depend on the wire length $(\propto 1 / L)$. We systematically measured the length dependence of the low-voltage, temperatureindependent structures; the results are presented in Fig. 12. The positions of the structures do not depend on the wire lengths for suspended or nonsuspended devices. The positions are given by the integer multiples of the same voltage, $V \approx 24 \mu \mathrm{V}$.

The fact that the positions of the temperature-independent structures are the same in different junctions makes it unlikely that they are related to external electromagnetic resonances, but rather result from some general intrinsic mechanism. To get a better insight into the origin of the temperature-independent subgap structures, we analyzed the shape of the IVC; see Fig. 13. In all investigated junctions, the IVC have a staircase
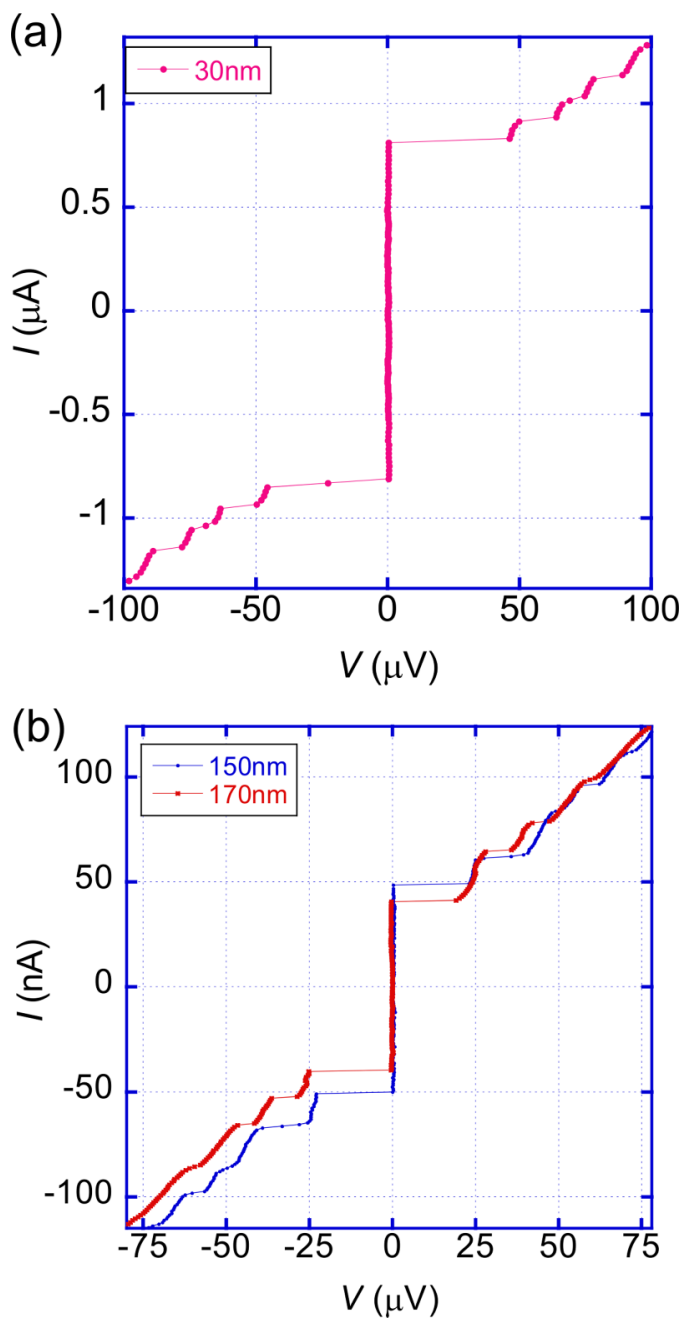

FIG. 13. (Color online) Current-voltage characteristics at low voltages for device $A_{1}$ (length $L=30 \mathrm{~nm}$ and normal-state resistance $R_{n}=0.16 \mathrm{k} \Omega$ ). The device shows successive voltage jumps with the resistance continuously increasing just after each voltage jumps. (b) Similarly, the current-voltage characteristics for somewhat longer devices, $B_{5 a}$ and $B_{5 b}(L=150$ and $170 \mathrm{~nm})$, show similar voltage steps at low voltages. shape and consist of a number of successive voltage steps. Between the steps, the current continuously grows with the differential resistance increasing after every step. Such a behavior may be explained by successive emergence of normally conducting domains in the wire as soon as the current exceeds the critical value. This picture closely resembles the resistive states in superconducting whiskers containing phase slip centers (PSC) [21,22]. Although one cannot in a straightforward way extend the PSC scenario in truly superconducting whiskers [45] to the proximity-induced superconductivity in nanowires, one cannot exclude the possibility of the formation of some kind of spatially inhomogeneous resistive state in the proximity region.

\section{GATE DEPENDENCE}

In this section, we investigate the gate dependence of the IVC in the superconducting state of suspended devices of type $C$ shown in Fig. 1(c) and in Table I. The data presented in the previous sections are obtained at the zero gate voltage for the conduction regime with multiple open conducting channels. Here we discuss the change of the IVCs in this regime with variation of the gate voltage. An opposite, few-channel transport regime at large negative gate voltage, showing quantization of the normal conductance and the Josephson critical current, was investigated in Ref. [11].

In our device, the gate voltage controls the local carrier concentration in the nanowire and thereby affects the strength of the proximity effect. Due to a strong capacitive coupling of the gate to the wire, this variation is significant, allowing us to observe a crossover from the SNS- to SIS-type regime of the current transport at low temperature. According to the theory $[30,46]$, the IVC of the transparent wire should exhibit, besides a large Josephson current, a large excess current both in

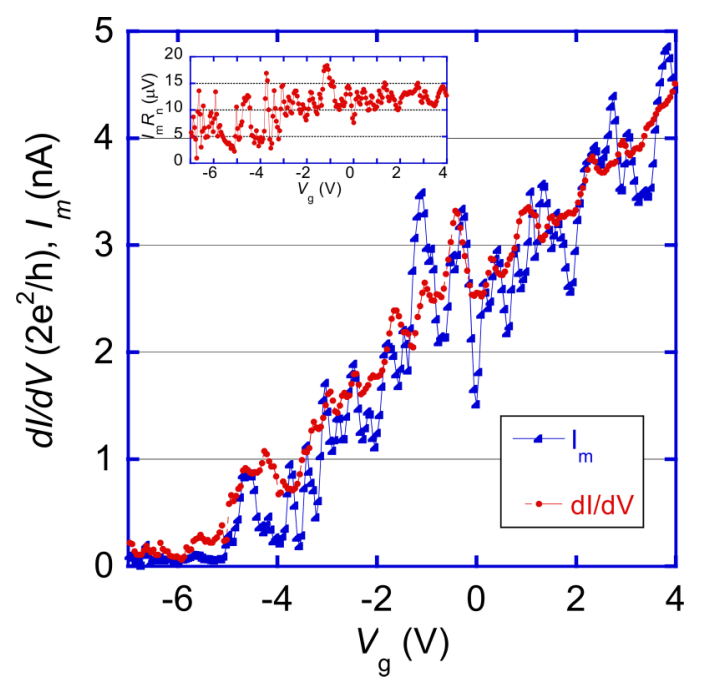

FIG. 14. (Color online) Normal-state conductance and maximum Josephson current as a function of a local-gate voltage for device $C_{11}$. After opening of the first conducting channel at $V_{g} \approx-5 \mathrm{~V}$, the overall conductance and critical current increase linearly with the gate voltage. Inset: the $I_{m} R_{n}$ product as a function of gate voltage. The constant value indicates that the maximum current is correlated with the normal-state conductance. 


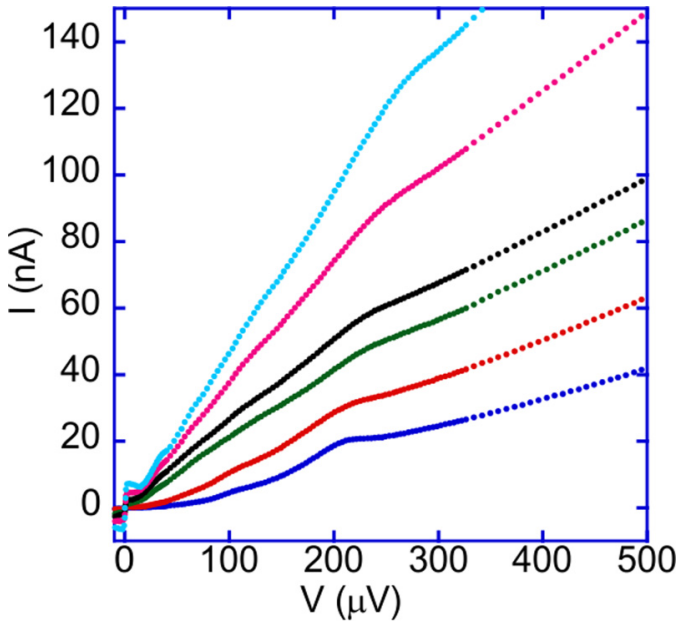

FIG. 15. (Color online) Current-voltage characteristics for device $C_{12}$ of length $L=200 \mathrm{~nm}$ for different gate voltages, $V_{g}=$ $-2.16,-1.86,-1.64,-1.40,-0.93,0.92 \mathrm{~V}$ (from bottom to top). The IVC exhibit crossover from the tunneling regime, with small subgap current and negative excess current, to the SNS regime, with enhanced subgap conductance and positive excess current.

the subgap voltage region and at the large voltage. On the other hand, more resistive wires should exhibit a small Josephson current, a suppressed subgap current, and a crossover to deficit (negative excess) current at large voltage.

The dependence of the maximum Josephson current on the gate voltage is shown in Fig. 14 for the suspended device $C_{11}(L \approx 150 \mathrm{~nm})$. The change of the maximum current (blue lines) varies in sync with the change of the differential conductance (red lines). Owing to the $n$-type nature of the nanowires, the conductance and the maximum Josephson current are strongly suppressed at large negative gate voltages, $V_{g}<-5$ V. Changing the gate voltage towards positive values results in linear increase of the averaged conductance and maximum Josephson current, with the latter reaching the value of $I_{m}=4$ $\mathrm{nA}$ at $V_{g}=3 \mathrm{~V}$. Simultaneously, the $I_{c} R_{n}$ product saturates at the value $I_{m} R_{n}=12.5 \mu \mathrm{V}$, and remains constant over a wide range of gate voltages, as shown in the inset in Fig. 14.

In Fig. 15, we present a set of IVCs for the suspended device $C_{12}\left(L \approx 200 \mathrm{~nm}\right.$ and $R_{n}=3.6 \mathrm{k} \Omega$ ) for gate voltages ranging from $V_{g}=-2.16$ to $V_{g}=+0.92 \mathrm{~V}$. At large positive gate voltage, i.e., at large conductance, the IVC shows significant excess current and enhanced subgap conductance, indicating a highly transmissive SNS regime. In the opposite limit of large negative gate voltage (small conductance), the IVC has a typical form for SIS tunnel junctions with negative excess current [39] and strongly suppressed subgap conductance. The suppression of the subgap conductance is explained by the small probability of MAR processes at small voltage, which scales with $\mathcal{D}^{n}$, where $n=2 \Delta / \mathrm{eV}$ is the number of Andreev reflections. In the tunneling regime with small $\mathcal{D} \ll 1$, the subgap conductance is exponentially small. At the intermediate gate voltages, the device exhibits continuous crossover between these two regimes, in accordance with the theoretical predictions for contacts with varying transparency $[30,46]$.

\section{CONCLUSION}

We have investigated, both experimentally and theoretically, the proximity effect in InAs nanowires connected to superconducting electrodes. We have fabricated and investigated a large number of nanowire devices with suspended gate-controlled nanowires and nonsuspended nanowires, with a broad range of lengths and normal-state resistances. We measured current-voltage characteristics and analyzed their main features: the Josephson current, excess current, and subgap current as functions of length, temperature, magnetic field, and gate voltage, and compared them with theory. The devices show reproducible resistance per unit length and highly transmissive interfaces. The measured superconducting characteristics are consistent and agree reasonably well in most cases with theoretically computed values. The maximum Josephson current for a short-length device, $L=30 \mathrm{~nm}$, exhibits a record high magnitude of $800 \mathrm{nA}$ at low temperature that comes close to the theoretically expected value. The maximum Josephson current in other devices is typically reduced compared to the theoretical values. The measured excess current in most of the devices is consistent with the normal resistance data and agrees well with the theory. The subgap current shows a large number of structures; some of them are identified as subharmonic gap structures generated by MAR. The other structures, detected in both suspended and nonsuspended devices, have the form of the voltage steps at voltages that are independent of either the superconducting gap or the length of the wire. By varying the gate voltage in suspended devices, we were able to observe a crossover from typical tunneling transport, with suppressed subgap current and negative excess current at large negative gate voltage, to pronounced SNS-type behavior, with enhanced subgap conductance and large positive excess current at large positive gate voltage.

\section{ACKNOWLEDGMENTS}

We acknowledge fruitful discussions with Lars Samuelson, Christopher Wilson, Thilo Bauch, and Jonas Bylander. The work was supported by the Swedish Research Council and the Wallenberg Foundation. H.Q.X. also acknowledges the National Basic Research Program of the Ministry of Science and Technology of China (Grants No. 2012CB932703 and No. 2012CB932700).
[1] L. Samuelson, Mater. Today 6, 22 (2003).

[2] C. Thelander, P. Agarwal, S. Brongersma, J. Eymery, L. Feiner, A. Forchel, M. Scheffler, W. Riess, B. Ohlsson, U. Gsele, and L. Samuelson, Mater. Today 9, 28 (2006).

[3] Y. Li, F. Qian, J. Xiang, and C. M. Lieber, Mater. Today 9, 18 (2006).
[4] S. A. Dayeh, Semicond. Sci. Technol. 25, 024004 (2010).

[5] I. van Weperen, S. R. Plissard, E. P. A. M. Bakkers, S. M. Frolov, and L. P. Kouwenhoven, Nano Lett. 13, 387 (2013).

[6] S. Abay, D. Persson, H. Nilsson, H. Q. Xu, M. Fogelström, V. Shumeiko, and P. Delsing, Nano Lett. 13, 3614 (2013). 
[7] T. S. Jespersen, M. L. Polianski, C. B. Sørensen, K. Flensberg, and J. Nygård, New J. Phys. 11, 113025 (2009).

[8] J. Xiang, A. Vidan, M. Tinkham, R. M. Westervelt, and Charles M. Lieber, Nat. Nanotechnol. 1, 208 (2006).

[9] Y.-J. Doh, J. A. van Dam, A. L. Roest, E. P. A. M. Bakkers, L. P. Kouwenhoven, and S. De Franceschi, Science 309, 272 (2005).

[10] H. A. Nilsson, P. Samuelsson, P. Caroff, and H. Q. Xu, Nano Lett. 12, 228 (2012).

[11] S. Abay, H. Nilsson, F. Wu, H. Xu, C. Wilson, and P. Delsing, Nano Lett. 12, 5622 (2012).

[12] T. Nishio, T. Kozakai, S. Amaha, M. Larsson, H. A. Nilsson, H. Q. Xu, G. Zhang, K. Tateno, H. Takayanagi, and K. Ishibashi, Nanotechnology 22, 445701 (2011).

[13] Y.-J. Doh, S. D. Franceschi, E. P. A. M. Bakkers, and L. P. Kouwenhoven, Nano Lett. 8, 4098 (2008).

[14] L. Hofstetter, S. Csonka, J. Nygård, and C. Schönenberger, Nature (London) 461, 960 (2009).

[15] V. Mourik, K. Zuo, S. M. Frolov, S. R. Plissard, E. P. A. M. Bakkers, and L. P. Kouwenhoven, Science 336, 1003 (2012).

[16] M. T. Deng, C. L. Yu, G. Y. Huang, M. Larsson, P. Caroff, and H. Q. Xu, Nano Lett. 12, 6414 (2012).

[17] A. Das, Y. Ronen, Y. Most, Y. Oreg, M. Heiblum, and H. Shtrikman, Nat. Phys. 8, 887 (2012).

[18] I. Kulik and A. Omel'yanchuk, Zh. Exp. Teor. Fiz. Pis. Red. 21, 216 (1975) [Sov. Phys. JETP Lett. 21, 96 (1975)].

[19] T. Klapwijk, G. Blonder, and M. Tinkham, Physica B+C 109, 1657 (1982).

[20] A. Kretinin, A. Das, and H. Shtrikman, arXiv:1303.1410.

[21] J. Meyer and G. Minnigerode, Phys. Lett. A 38, 529 (1972).

[22] W. Skocpol, M. Beasley, and M. Tinkham, J. Low Temp. Phys. 16, 145 (1974).

[23] B. Ohlsson, Physica E 13, 1126 (2002).

[24] H. A. Nilsson, T. Duty, S. Abay, C. Wilson, J. B. Wagner, C. Thelander, P. Delsing, and L. Samuelson, Nano Lett. 8, 872 (2008).

[25] D. B. Suyatin, C. Thelander, M. T. Björk, I. Maximov, and L. Samuelson, Nanotechnology 18, 105307 (2007).

[26] S. Datta, Electronic Transport in Mesoscopic Systems (Cambridge University Press, Cambridge, 1997).
[27] P. Samuelsson, A. Ingerman, G. Johansson, E. V. Bezuglyi, V. S. Shumeiko, G. Wendin, R. Kürsten, A. Richter, T. Matsuyama, and U. Merkt, Phys. Rev. B 70, 212505 (2004).

[28] A. Furusaki, H. Takayanagi, and M. Tsukada, Phys. Rev. B 45, 10563 (1992).

[29] G. Arnold, J. Low Temp. Phys. 68, 1 (1987).

[30] E. N. Bratus', V. S. Shumeiko, and G. Wendin, Phys. Rev. Lett. 74, 2110 (1995).

[31] D. Averin and A. Bardas, Phys. Rev. Lett. 75, 1831 (1995).

[32] J. C. Cuevas, A. Martín-Rodero, and A. Levy Yeyati, Phys. Rev. B 54, 7366 (1996).

[33] The crossover from the diffusive short- to long-junction regime could alternatively be modeled with a theory developed in Ref. [39].

[34] J. C. Cuevas and M. Fogelström, Phys. Rev. B 64, 104502 (2001).

[35] M. Eschrig, Phys. Rev. B 80, 134511 (2009).

[36] A. V. Zaitsev, Zh. Exp. Teor. Fiz 86, 1742 (1984) [Sov. Phys. JETP 59, 1015 (1984)].

[37] V. S. Shumeiko, E. N. Bratus, and G. Wendin, Low Temp. Phys. 23, 181 (1997).

[38] J. C. Cuevas, J. Hammer, J. Kopu, J. K. Viljas, and M. Eschrig, Phys. Rev. B 73, 184505 (2006).

[39] E. V. Bezuglyi, E. N. Bratus', and V. S. Shumeiko, Phys. Rev. B 83, 184517 (2011)

[40] J. M. Martinis and H. Grabert, Phys. Rev. B 38, 2371 (1988).

[41] V. Ambegaokar and B. I. Halperin, Phys. Rev. Lett. 22, 1364 (1969).

[42] K. Bladh, D. Gunnarsson, E. Hürfeld, S. Devi, C. Kristoffersson, B. Smålander, S. Pehrson, T. Claeson, P. Delsing, and M. Taslakov, Rev. Sci. Instrum. 74, 1323 (2003).

[43] M. Y. Kupriyanov, A. Brinkman, A. A. Golubov, M. Siegel, and H. Rogalla, Physica C 326, 16 (1999).

[44] G. E. Blonder, M. Tinkham, and T. M. Klapwijk, Phys. Rev. B 25, 4515 (1982).

[45] V. Galaiko and N. Kopnin, in Nonequilibrium Superconductivity, edited by D. Langenberg and A. Larkin (North-Holland, Amsterdam, 1986).

[46] A. Ingerman, G. Johansson, V. S. Shumeiko, and G. Wendin, Phys. Rev. B 64, 144504 (2001). 